\title{
0 perfil oftalmológico dos candidatos à carteira de motorista na cidade de Pelotas - RS
}

\author{
Ophthalmological profile of the candidates fordriving license in Pelotas - RS
}

\author{
Wladimir Ribeiro Duarte ${ }^{1}$ \\ Maria Claudete Ribeiro Duarte ${ }^{2}$ \\ Carla Paranhos ${ }^{3}$ \\ Jussara Ribeiro Duarte Bocaccio ${ }^{2}$ \\ Francisco José Lima Bocaccio ${ }^{4}$ \\ Guaraciaba Ribeiro Duarte Sousa ${ }^{5}$ \\ Márcia Ribeiro Duarte ${ }^{6}$
}

\section{RESUMO}

Objetivo: Estudar a acuidade visual dos candidatos a condutores de veículos da cidade de Pelotas e sua associação com as características sociodemográficas dos candidatos. Métodos: Foram examinados 1.010 candidatos a condutores em um serviço médico autorizado pelo Detran. Avaliou-se a acuidade visual, com e sem correção, bem como a sua relação com a visão de cores, sexo, idade e profissão dos entrevistados, por meio da análise de freqüências e de teste para associação. Resultados: Deste contingente $73,0 \%$ eram do sexo masculino, 65,0\% tinham idade entre 18 e 45 anos, $51,4 \%$ ocupavam o setor de serviços e $25,0 \%$ utilizavam meio de correção visual. Sob o ponto de vista oftalmológico, $0,5 \%$ dos entrevistados não poderiam dirigir veículos automotores e $4,7 \%$ só poderiam obter habilitação para condução veicular, nas categorias A e B. Mostrou-se associada à acuidade visual baixa a maior longevidade. Conclusão: Baseado nos resultados, sugere-se menor intervalo de tempo para reavaliação visual dos motoristas das categorias C, D e E.

Descritores: Acuidade visual; Testes visuais; Transtornos da visão/diagnóstico; Exame para habilitação de motoristas; Percepção de cores; Fatores socioeconômicos; Defeitos da visão de cores; Fatores sexuais

\section{INTRODUÇÃOO}

Aspectos oftalmológicos de diferentes populações de motoristas condutores de de veículos pesados no território nacional foram motivos de estudos por Brandão et al., onde os autores constataram serem necessárias modificações e avanços na legislação brasileira de trânsito, em vista da gravidade atual dos problemas de tráfego ${ }^{(1)}$.

De acordo com a legislação de trânsito, para estar apto para conduzir veículos automotores nas categorias A e B, sob o ponto de vista de acuidade visual e visão de cores, é necessário: visão de 0,66 nos dois olhos, todavia, no caso de visão inferior a 0,66 em um dos olhos, a visão no melhor olho deverá ser igual ou superior a 0,80 (Tabela de Snellen). Exige-se do candidato a identificação das cores vermelha, amarela e verde ${ }^{(2)}$.

Convém observar que para os portadores de visão monocular (acuidade 0 - zero) ou visão baixa em qualquer olho (inferior a 0,5 ), está vedada a atividade remunerada vinculada à direção de veículos automotores, conseqüentemente para as categorias C, D e E não se aceita visão inferior a 0,66 em qualquer olho(2).

Para manter a habilitação já adquirida, a legislação de trânsito exige que a cada 5 anos haja a repetição de alguns testes e entre estes, inclui-se o de acuidade visual ${ }^{(2)}$.

As pesquisas sobre a higidez visual dos condutores de veículos auto- 
motores são de extrema relevância, haja vista, que a visão corresponde por $95 \%$ do ato de dirigir ${ }^{(1,3)}$, bem como, somente no ano de 1988 ocorreram 30.994 mortes anuais no Brasil decorrentes de acidentes de trânsito, o que se leva a acreditar que as deficiências visuais respondem por uma fração importante destes desfechos ${ }^{(4-5)}$.

A presente pesquisa, foi realizada em um serviço médico autorizado pelo DETRAN para a realização da avaliação de saúde dos candidatos a carteira de habilitação ou sua renovação.

Neste estudo, verificou-se a acuidade visual de longe e o perfil social e demográfico dos candidatos (primeira habilitação ou renovação da carteira), através da análise da freqüência de: déficit visual, discromatopsias, uso de correção visual, ocupação, sexo e faixa etária, bem como a verificação de possível associação da acuidade visual com as variáveis: sexo, idade e correção visual.

\section{MÉTODOS}

Este estudo foi delineado e executado como transversal de base de serviços, onde os dados foram primários. Foi realizado na cidade de Pelotas - RS, uma cidade da região Sul do Brasil, com 307.667 habitantes onde $91,8 \%$ residem no meio urbano ${ }^{(6)}$.

Foram eleitos para o estudo todos os candidatos à carteira de habilitação ou à renovação da mesma, que possuíssem 18 ou mais anos de vida e que procurassem o referido serviço médico autorizado pelo DETRAN entre os meses de junho a setembro de 1997. Observa-se que nesta cidade e no mesmo período existiam mais dois serviços médicos habilitados para a avaliação de saúde para a carteira de habilitação.

Para mensurar a acuidade visual foi utilizada a tabela de Snellen, além de 1500 questionários padronizados e pré-codificados para registro das variáveis.

A acuidade visual foi registrada medindo-se individualmente cada olho com a tabela de optotipos de Snellen (marca Xenônio ${ }^{\circledR}$ ) com iluminação própria para a distância de 6 metros (padronizou-se que o primeiro olho a ser medido fosse o direito) e a verificação da condição visual para cores foi testada na própria tabela utilizando fontes luminosas nas cores: vermelha, verde e amarela.

O critério utilizado para definir acuidade visual baixa (AVB) para longe foi que o entrevistado tivesse acuidade visual inferior a 0,66 a seis metros de distância, ao menos em um olho ${ }^{(7)}$.

Para os cálculos de verificação de associação, utilizou-se dois diferentes pontos de corte para acuidade visual baixa considerando o olho de menor visão: um ponto de corte abaixo de 0,66 e o outro abaixo de 0,8 .

Para os indivíduos que utilizavam correção visual para longe (óculos ou lentes de contato), a acuidade visual foi medida com o uso da correção.

Os questionários continham as seguintes variáveis: nome por extenso do candidato, o sexo, a idade em anos completos, o tipo de ocupação dividida nas seguintes categorias: (1) agricultura ou exploração primária, (2) serviços, (3) comércio,
(4) indústria, (5) aposentados ou estudantes e (6) desempregados, além do uso de algum meio de correção visual.

Observa-se que os dados foram coletados por um único médico, oftalmologista, sendo a realização dos testes executados pelo mesmo profissional.

As informações obtidas foram digitadas duas vezes no programa EPI INFO por 2 pessoas diferentes, onde foi aplicado um protocolo de consistência das digitações. Para a verificação de associação, utilizou-se o teste do Qui-quadrado de Pearson e para a medida de efeito utilizou-se a razão de prevalências, sendo as análises feitas com o uso dos programas de estatística SPSS 8.0 e EPI INFO 6.02(8-9).

\section{RESULTADOS}

Neste estudo 1010 pessoas foram entrevistadas, havendo duas perdas para uma variável. Ao considerar as categorias mais prevalentes de cada variável (Tabela 1), o perfil dos entrevistados foi: ser do sexo masculino $(73,0 \%)$, ter idade entre 18 e $45 \operatorname{anos}(65,0 \%)$, ter atividade ocupacional vinculada ao setor de serviços $(51,4 \%)$, não ser portador de discromatopsia $(99,9 \%)$ e não ser usuário de meio de correção visual $(75,0 \%)$.

Quanto à condição visual (Gráfico 1), 97,6\% das pessoas possuíam visão igual ou superior a 0,66 para o olho direito e 96,8 \% para o olho esquerdo. Neste estudo, 94,9\% dos entrevistados apresentaram visão igual ou superior a 0,66 em ambos os olhos (AO) e apenas 5 pessoas tiveram visão inferior a 0,66 (AO), sendo 2 pessoas do sexo feminino e 3 do sexo masculino (Tabela 2). Um indivíduo não identificou a cor vermelha.

\begin{tabular}{|c|c|c|c|}
\hline Variável & Freqüência & $\begin{array}{c}\text { Percentual } \\
(\%)\end{array}$ & $\begin{array}{l}\text { AVB } \\
(\%)\end{array}$ \\
\hline \multicolumn{4}{|l|}{$\operatorname{Sexo}(N=1010)$} \\
\hline Masculino & 737 & 73,0 & 4,6 \\
\hline Feminino & 273 & 27,0 & 6,6 \\
\hline \multicolumn{4}{|l|}{ Idade $(N=1010)$} \\
\hline $18-45$ anos & 657 & 65,0 & 5,6 \\
\hline 45 anos ou mais & 353 & 35,0 & 4,2 \\
\hline \multicolumn{4}{|l|}{ Profissão $(\mathrm{N}=1008)$ ** } \\
\hline Agropecuária / extrativismo & 49 & 4,9 & 4,1 \\
\hline Comércio & 131 & 13,0 & 1,5 \\
\hline Indústria & 20 & 2,2 & 0,0 \\
\hline Serviços & 519 & 51,4 & 5,6 \\
\hline Estudantes & 114 & 11,3 & 7,0 \\
\hline Outros & 173 & 17,1 & 6,4 \\
\hline \multicolumn{4}{|l|}{ Discromatopsia ( $N=1010)$} \\
\hline Não & 1008 & 99,9 & 5,1 \\
\hline $\operatorname{Sim}$ & 1 & 0,1 & 0,0 \\
\hline \multicolumn{4}{|l|}{ Correção ocular ( $N=1010)$} \\
\hline Não & 757 & 75,0 & 4,8 \\
\hline Sim & 253 & 25,0 & 6,3 \\
\hline
\end{tabular}




\section{OLHODIREITO}

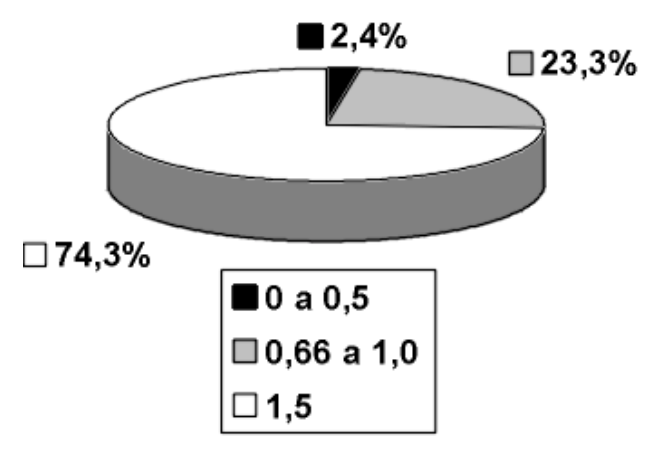

\section{OLHO ESQUERDO}

\section{$74,4 \%$}
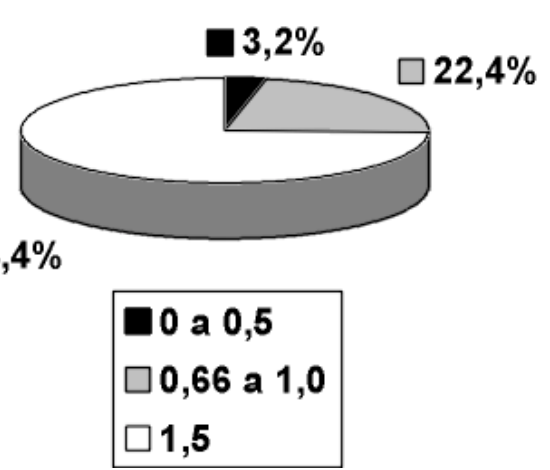

Gráfico 1 - Gráfico dos percentuais dos níveis de acuidade visual, considerando cada olho separadamente. Pelotas, 2001

Utilizando o teste de associação do $\chi^{2}$ (Qui-quadrado de Pearson), não se obteve associação significativa para um nível de significância de 5\% entre acuidade visual baixa (visão inferior a 0,66 em pelo menos um olho) e as variáveis: sexo, idade e uso de correção visual. Porém ao se considerar como acuidade visual baixa a visão inferior a 0,8 em pelo menos um olho (Tabela $3)$, observou-se associação significativa $(\mathrm{p}<0,05)$ com a maior longevidade, resultando em um risco relativo de 2,03.

\section{DISCUSSÃO}

Pelo fato deste estudo ser transversal e de base de serviço médico, tem como limitações a possibilidade de ocorrerem vieses de seleção e de informação, não podendo descrever causalidade, além de estar sujeito a fatores de confusão e interação ${ }^{(10)}$.

Nesta investigação a maior parte dos candidatos era do sexo masculino $(72,5 \%)$ e eram pessoas jovens $(52,7 \%)$. Este predomínio pode estar refletindo a influência dos fatores sociais e demográficos nas diferentes oportunidades de inserção socioeconômica, onde jovens do sexo masculino podem ser mais motivados a obter a carteira de habilitação ${ }^{(11)}$.

Em relação a discromatopsia, acredita-se que a baixa preva-

Tabela 2. Distribuição de freqüências de acuidade visual, considerando a visão nos dois olhos. Pelotas, 2001

\begin{tabular}{lcc} 
Variável & Freqüência & $\begin{array}{c}\text { Percentual } \\
(\%)\end{array}$ \\
Acuidade visual binocular (N=1010) & & \\
0,66 ou superior A O * & 958 & 94,9 \\
0,8 ou superior em um olho e 0,5 no outro & 10 & 1,0 \\
$\begin{array}{l}\text { 0,8 ou superior em um olho e } \\
\text { inferior a 0,5 no outro }\end{array}$ & 37 & 3,7 \\
$\begin{array}{l}\text { inferior a 0,66 A O * } \\
\text { * AO - ambos os olhos }\end{array}$ & 5 & 0,5 \\
\hline
\end{tabular}

lência encontrada nesta amostra $(0,1 \%)$ deva-se à baixa sensibilidade do método utilizado, pois de acordo com os dados da literatura, a prevalência mundial quando medida com a tabela de Ishihara encontra-se em torno de $8 \%$ na população masculina e $0,4 \%$ na população feminina ${ }^{(12)}$, contudo a legislação atual veda a licença para dirigir quando há deficiência visual para as cores vermelha, verde e amarela ${ }^{(2)}$.

Supõe-se que a reduzida proporção de indivíduos $(0,5 \%)$ que apresentaram visão inadequada para qualquer categoria de habilitação ao trânsito na ocasião do estudo, possa ter resultado da auto-seleção dos candidatos, ou seja, aqueles que possuíam boa visão e usavam correção visual adequada procuraram o serviço, ao contrário daqueles com visão inadequada e sem meio corretivo. Entretanto, se considerarmos o critério visual para a habilitação nas categorias C, D e E, outros 4,7\% dos entrevistados não estariam capacitados, onde apenas $1,0 \%$ foram aptos para as categorias A e B com possibilidade de ter atividade remunerada vinculada à direção de automotores.

Tabela 3. Associação para acuidade visual baixa - AVB*. Pelotas, 2001

\begin{tabular}{|c|c|c|c|c|}
\hline Variável & $\begin{array}{c}\text { Freqüência } \\
\%\end{array}$ & $\begin{array}{l}\text { Prevalência } \\
\text { de AVB \% }\end{array}$ & $\begin{array}{l}\text { Razão de } \\
\text { prevalências } \\
\text { (IC } 95 \text { \%) }\end{array}$ & Valor-p* \\
\hline \multicolumn{4}{|l|}{ Idade (anos) } & \multirow[t]{3}{*}{$<0,001$} \\
\hline 18 a 45 & 65,0 & 8,2 & 1 & \\
\hline 46 ou mais & 35,0 & 16,7 & $2,03(1,44-2,87)$ & \\
\hline \multicolumn{4}{|l|}{ Sexo } & \multirow[t]{3}{*}{0,6} \\
\hline masculino & 73,0 & 10,9 & 1 & \\
\hline feminino & 27,0 & 12,1 & $1,11(0,76-1,73)$ & \\
\hline \multicolumn{4}{|c|}{ Correção visual } & \multirow[t]{3}{*}{0,53} \\
\hline não & 75,0 & 10,8 & 1 & \\
\hline $\operatorname{sim}$ & 25,0 & 12,3 & $1,13(0,77-1,67)$ & \\
\hline
\end{tabular}


Não foi encontrada associação significativa entre o sexo, idade e uso de meio corretivo com baixa acuidade visual dos candidatos (considerando a acuidade visual inferior a 0,66 no olho de menor visão).

Alternativamente, ao se considerar como acuidade visual baixa os valores de acuidade visual inferiores a 0,8 , encontrou-se forte associação com maior longevidade, fato sustentado por plausibilidade biológica, indicando que quanto maior a idade, maior será a chance de ser acometido por patologias que interfiram com a visão ${ }^{(13)}$. Vários estudos evidenciam este achado, onde a longevidade é diretamente proporcional aos agravos visuais $^{(14)}$.

Em acordo com os dados desta investigação sugere-se que deva haver um maior debate, por parte da classe oftalmológica, sobre a influência da idade na obtenção da licença para dirigir, pois talvez exista a necessidade de se realizar testes visuais mais freqüentes do que a exigida pela legislação atual para os portadores de habilitação para as categorias $\mathrm{C}, \mathrm{D}$ e E. Corroborando esta hipótese, existem evidências do maior envolvimento dos motoristas de carga com acidentes mais graves $^{(1,4)}$, bem como pelos aumentos crescentes nas frotas de automotores nas vias nacionais ${ }^{(1)}$.

No Brasil, onde os acidentes automobilísticos assumem uma posição de destaque no preocupante aumento da mortalidade por causas externas ${ }^{(5)}$, as ações preventivas com enfoque para a saúde, principalmente do sistema visual dos condutores de veículos devem ser intensificadas, priorizando a constante qualificação dos profissionais responsáveis pelas triagens de cunho médico ${ }^{(12,15)}$.

\section{CONCLUSÃO}

Acredita-se que este estudo avança ao quantificar a medida de acuidade visual dos candidatos a condutores de trânsito, bem como ao procurar caracterizar possíveis fatores relacionados à baixa condição visual nesta população. Os dados deste trabalho não nos permitem extrapolar para a população em geral, porém acreditamos que outros estudos congêneres possam somar-se a este e, com maior amostragem, permitir fazer-se ilações mais consistentes quanto a este importante assunto de saúde pública.

\section{ABSTRACT}

Purpose: To study visual acuity of the candidates for driving licences in the city of Pelotas and its relationship with sociodemographic characteristics of the candidates. Methods: One thousand and ten candidates for driving license were examined in a Detran approved medical service. To check the social, demographic and visual characteristics of candidates, visual acuity, with or without correction, as well as its relation to color, gender, age and profession of the interviewed persons was evaluated, through analysis of frequencies and association. Results: In this study $72.5 \%$ were males, $33.8 \%$ were between 18 and 45 years old, $52.0 \%$ were in the service sector and $25.4 \%$ used some instrument of visual correction, $0.5 \%$ of the interviewed from the ophthalmological viewpoint, would not be able to drive automotor vehicles and $4.7 \%$ should only would get a driving license in the A and B categories. A low visual acuity has been associated with a higher longevity. Conclusion: Based on the results, a shorter time period for visual reavaluation of the $\mathrm{C}, \mathrm{D}$ and $\mathrm{E}$ categories drivers is suggest.

Keywords: Visual acuity; Vision tests; Vision disorders/diagnosis; Automobile driver examination; Color perception; Socioeconomic factors; Color vision defects; Sex factors

\section{REFERÊNCIAS}

1. Brandão EO, Oliveira NM, Pereira MVC, Maia NCF, Pereira GC. Acuidade visual de motoristas de veículos pesados numa rodovia de grande circulação:problemática e sugestões. Arq Bras Oftalmol 1995;58:121-6.

2. Brasil. Conselho Nacional de Trânsito. Resolução $N^{0} 80$, de 19 de Novembro de 1988. Dispõe sobre os exames de aptidão física e mental e os exames de avaliação psicológica. Brasília, CONTRAN - Conselho Nacional de Trânsito 1988. [citado em 2002 Set. 12], Disponível em: URL: http://www.mj.gov.br/ Contran/resolu.htm.

3. Ramos ARB, Zaniolo S, Matsumoto LH, Ramos CCB, Ferreira RL, Santos $\mathrm{L}$ et al. Causas de reprovação no teste de acuidade visual do Departamento de Trânsito do Paraná. Arq Bras Oftalmol 1994;57:305-9.

4. Owsley C, McGwin Jr.G, Ball K. Vision impairmente, eye diseases, and injurious motor vehicle crashes in the elderly. Ophthalmic Epidemiol 1988; 5:101-13.

5. Brasil. Ministério da Saúde. Datasus. C.9 Taxa de mortalidade específica por causas externas: número de acidentes por transportes. [citado em 2002 Set. 12], Disponível em: URL: http://tabnet.datasus.gov.br/cgi/tabcgi.exe?idb2001/ c09.def.

6. Instituto Brasileiro de Geografia e Estatística. IBGE. Contagem da população 1996. Resultados relativos à situação de unidade domiciliar da população. Rio de Janeiro: IBGE; 1997.

7. Concilium Ophtalmologicum Universale.Comitê de funções visuais. Medida padrão da acuidade visual. Arq Bras Oftalmol 1988;51:203-13.

8. EPI INFO, 1997. Epi Info Version 6.02. A World Processing, Database, and Statistics System for Epidemiology on Microcomputers. Geneva: World Health Organization.

9. SPSS for Windows 1994. Statistical Package for the Social Sciences. Release 6.1 Chicago: SPSS Inc.

10. Pereira MG. Estrutura, vantagens e limitações dos principais estudos. In: Pereira MG. Epidemiologia: teoria e prática. Rio de Janeiro, Guanabara Koogan; 1995. p.298-99.

11. Olinto MTA. Reflexões sobre o uso do conceito de gênero e/ou sexo na epidemiologia: um exemplo nos modelos hierarquizados de análise. Rev Bras Epidemiol 1998;1:161-9.

12. Vaughan D, Asbury T. Oftalmologia geral. 3 $3^{\mathrm{a}}$ ed. São Paulo: Atheneu; 1983. $191 \mathrm{p}$.

13. Fletcher H, Fletcher SW, Wagner EH. Casualidade. In: Fletcher H, Fletcher SW. Epidemiologia clínica: elementos essenciais. $3^{\text {a }}$ ed. Porto Alegre: Artes Médicas; 1996. p.251-52.

14. Hankinson SE. The epidemiology of age related cataract. In: Albert DM, Jakobiec FA. Principles and practice of ophthalmology: basic sciences. Philadelphia: W. B. Saunders; 1994. p.1256-57.

15. Sotero SM, Duncan BB. Aspectos das condições de saúde da população brasileira. In: Duncan BB, Schmidt MI, Giugliani ERJ. Medicina ambulatorial: condutas clínicas em atenção primária. 2a ed. Porto Alegre: Artes Médicas; 1996. p.327-32. 